\title{
Solvothermal synthesis of polyazomethine microspheres by Pickering emulsion templates and their transformation into complex microtubes and anisotropic hollow spheres enabled by dynamic imine chemistry
}

\begin{abstract}
Zihua Chen, Yi Jiang, Lu Chen, Wei Huang, Xiang Li, Xiaojuan Li and Xikui Liu
Dynamic covalent chemistry has attracted much attention in recent decades. Here we report a facile one-pot solvothermal synthesis of anisotropic polyazomethine particles using Pickering emulsions formed in situ as templates. An unexpected morphological transformation from nanoparticles to anisotropic particles and finally to complex anisotropic yolk-shell structures was observed. The underlying mechanism is a controlled chemical etching process enabled by the dynamic imine bonding and the inhomogeneous nature of the resulting anisotropic particles. The generalizability of this process was further proved by fine tuning the initial solvent composition, through which an interesting morphological transformation from hollow spheres to wormlike tubes and finally to microtubes with a smooth surface was observed, thus providing a novel template-free strategy for the synthesis of polymeric materials with complex hollow interiors, which has rarely been reported in polymeric systems.
\end{abstract}

Polymer Journal (2013) 45, 1087-1093; doi:10.1038/pj.2013.23; published online 13 March 2013

Keywords: anisotropic particles; dynamic imine chemistry; Pickering emulsion; polyazomethine; yolk-shell particles

\section{INTRODUCTION}

Anisotropic particles have attracted rapidly increasing attention in recent decades, mainly because of their interesting asymmetric features, which enable great potential in fields such as emulsion, phase-transfer catalysts and sensing devices. ${ }^{1-7}$ One approach generally used to synthesize anisotropic particles is the so-called phase interface method, which involves trapping the homogeneous particles at the interface of immiscible solvents to form a Pickering emulsion, then functionalizing the particles in one of the phases to achieve anisotropic structures; ${ }^{8-10}$ however, this process is generally tedious. Moreover, even though anisotropic particles based on aromatic polymers are clearly effective for many applications because of their excellent mechanical and thermal properties, because of their poor solubility, such anisotropic structures have rarely been reported.

Dynamic covalent chemistry involves the generation of molecular constituents through reversible covalent bonds whose unique dynamic nature offers great possibilities for the structure and morphology control of polymers. ${ }^{11-13}$ For example, Yaghi and colleagues $^{14-16}$ overcame the long-standing 'crystallization problem' of three-dimensional polymer networks using dynamic boronate bonding, whereas Kubo and colleagues ${ }^{17}$ reported an interesting dynamic morphology transformation from microsphere to microribbon by taking advantage of dynamic covalent boronate ester bonding. Very recently, we found that the introduction of dynamic imine bonding could aid in the crystallization control of aromatic polymers. ${ }^{18}$ However, we believe that we are just beginning to understand the mechanism behind dynamic covalent chemistryenabled morphology control, and many interesting aspects remain largely unexplored.

With this in mind, here we report a facile one-pot solvothermal synthesis of anisotropic particles composed of fully conjugated aromatic polyazomethine networks using the Pickering emulsions formed in situ as templates. A completely unexpected morphological transformation from irregular nanoparticles to Christmas ball-like and finally to semi-hairy anisotropic particles was observed when tracing the solvothermal process. We believe that the mechanism is a controlled chemical etching process enabled by the unique $\mathrm{pH}$-sensitive nature of dynamic imine bonding and the inhomogeneous nature of the anisotropic particles. This argument is supported by the fact that the anisotropic particles gradually hollowed and transformed to complex anisotropic yolk shells and completely hollow spheres when they were further treated in a stronger acid to enhance the reversibility of dynamic imine bonding. The generalizability of 
this process was further proved by fine tuning the initial solvent composition, through which an interesting morphological transformation from hollow spheres to worm-like tubes and finally to microtubes with smooth surfaces was observed. Our results provide a powerful template-free approach to the fabrication of complex polymeric structures such as anisotropic yolk-shell particles, which, according to our knowledge, have not been reported in polymeric systems.

\section{EXPERIMENTAL PROCEDURE}

\section{Materials}

2,4,6-tris(4-aminophenyl)pyridine (TAPP), 5,10,15,20-tetrakis (4-aminophenyl)$21 \mathrm{H}, 23 \mathrm{H}$-porphine and tetrakis(4-aminophenyl) methane were synthesized using the reported procedure, and the details of the synthesis are described in the supporting information. 1,4-Terephthalaldehyde (TPA) was purchased from Sigma-Aldrich (Steinheim, Germany) and used as received. Other solvents were purchased from Chengdu Kelong chemicals (Chengdu, China) and purified using standard processes.

\section{Measurements \\ Scanning electron microscopy (SEM) was conducted with an Inspect F SEM at an accelerating voltage of $20 \mathrm{kV}$. Transmission electron microscopy observa- tions were performed with a JEM 100CX II transmission electron microscope (Jeol, Tokyo, Japan) at an accelerating voltage of $80 \mathrm{kV}$. The chemical structures were characterized by infrared spectra with a Nicolet 560 spectrometer (Nicolet, Madison, WI, USA). The crystalline structures were characterized by an x-pert pro MPD diffractometer using powder samples at $30 \mathrm{kV}$ and $15 \mathrm{~mA}$ with a scanning rate of $1^{\circ} \mathrm{min}^{-1}$.}

\section{Preparation of anisotropic polyazomethine particles}

A typical process was as follows: TAPP $(140.8 \mathrm{mg}, 0.40 \mathrm{mmol})$ and TPA $(80.4 \mathrm{mg}, 0.60 \mathrm{mmol})$ were placed in a teflon-lined autoclave containing $5.0 \mathrm{ml}$ of anhydrous dioxane and $5.0 \mathrm{ml}$ mesitylene, and after sonication for $30 \mathrm{~min}$, $1.0 \mathrm{ml}$ of $6 \mathrm{M}$ aqueous acetic acid was added. The autoclave was then transferred into an oven and reacted at designated temperatures for various times. After that, the resulting yellow polyazomethine products were collected by filtration and then dried in a vacuum.

Preparation of anisotropic yolk-shell particles and hollow spheres The anisotropic particles synthesized as above were placed in a teflon-lined autoclave containing $5.0 \mathrm{ml}$ anhydrous dioxane and $5.0 \mathrm{ml}$ glacial acetic acid. It is worth noting that the mixture solvent was homogeneous. The autoclave was then transferred into an oven and heated at $120^{\circ} \mathrm{C}$ for $48 \mathrm{~h}$ to partially cavitate the anisotropic particles, resulting in anisotropic yolk-shell structures. Additional treatment with a longer reaction time of $96 \mathrm{~h}$ completely cavitated the anisotropic particles and resulted in anisotropic hollow spheres.

\section{Preparation of hollow spheres and their transformation into microtubes}

A typical process was as follows: TAPP $(140.8 \mathrm{mg}, 0.40 \mathrm{mmol})$ and TPA $(80.4 \mathrm{mg}, 0.60 \mathrm{mmol})$ were placed in a teflon-lined autoclave containing $2.5 \mathrm{ml}$ of anhydrous dioxane and $7.5 \mathrm{ml}$ mesitylene, and after sonication for $30 \mathrm{~min}$, $1.0 \mathrm{ml}$ of $6 \mathrm{M}$ aqueous acetic acid was added. The autoclave was then transferred to an oven and reacted at designated temperatures for various times. A morphological transformation from a hollow sphere to a worm-like microtube and finally to a microtube with smooth surface was observed during the different reaction stages.

\section{RESULTS AND DISCUSSION}

For TPA and TAPP reacted in an immiscible mixture solution of mesitylene, 1,4-dioxane and aqueous acetic acid (5/5/1 vol), the polyazomethines that precipitated from the mixture solution immediately were irregular nanoparticles with diameters of approximately $100 \mathrm{~nm}$ (Figure 4a). In contrast to traditional Pickering emulsions, where the preformed particles are used as surfactants, we found that the polyazomethine nanoparticles formed in situ tended to aggregate at the interface of the immiscible solution to decrease the surface energy, thus forming a type of nanoparticle-stabilized water-in-oil Pickering emulsion. Upon further treatment by a solvothermal process for $1 \mathrm{~h}$, the irregular nanoparticles located at the oil/water interface of the Pickering emulsion transformed into regular microspheres with smooth surfaces (Figures 1 and $4 \mathrm{~b}$ ).

After solvothermal processing for $72 \mathrm{~h}$, the resulting yellow powder was separated and characterized. SEM clearly revealed that anisotropic particles with diameters of approximately $3 \mu \mathrm{m}$ were formed and that half of each particle's surface was relatively smooth and the other half was relatively rough (Figure $2 \mathrm{a}$ ). Decreasing the polymer concentration to $1 \%$ also resulted in uniform anisotropic particles that possessed a shorter, hairy nanostructure (Figure 2b). SEM also revealed that the obtained anisotropic particles were linked together and could not be broken into discrete individual anisotropic particles, even by sonification; this is believed to be caused by the strong chemical bonding between the contact surfaces of the resulting nanofibers.

To demonstrate the generalizability of this facile method of Pickering emulsion templates formed in situ, two additional amino monomers, tetrakis(4-aminophenyl)porphyrin and tetrakis(4-aminophenyl)methane, were used instead of TAPP to react with TPA. After the solvothermal process, anisotropic particles with diameters of several micrometers were again observed, but in this case, smooth anisotropic particles, rather than semi-hairy anisotropic structures, were obtained (Figures $3 \mathrm{a}$ and $\mathrm{b}$ ), thus confirming the generalizability of this Pickering emulsion template approach.

We quenched the solvothermal process at different stages to get a clearer picture of this facile-synthesis approach for anisotropic particles, and a completely unexpected and interesting continuous morphological transformation from irregular nanoparticles to Christmas ball-like and finally to uniform anisotropic microparticles was observed (Figure 4). The polyazomethines precipitated from the solution were irregular nanoparticles with diameters of approximately $100 \mathrm{~nm}$ (Figure 4a). These nanoparticles tended to aggregate at the interface of the immiscible solution to decrease the surface energy, thus forming a type of nanoparticle-stabilized water-in-oil Pickering emulsion. Upon additional treatment by a solvothermal process, the irregular nanoparticles located at the oil/water interface quickly transformed into regular microspheres with smooth surfaces

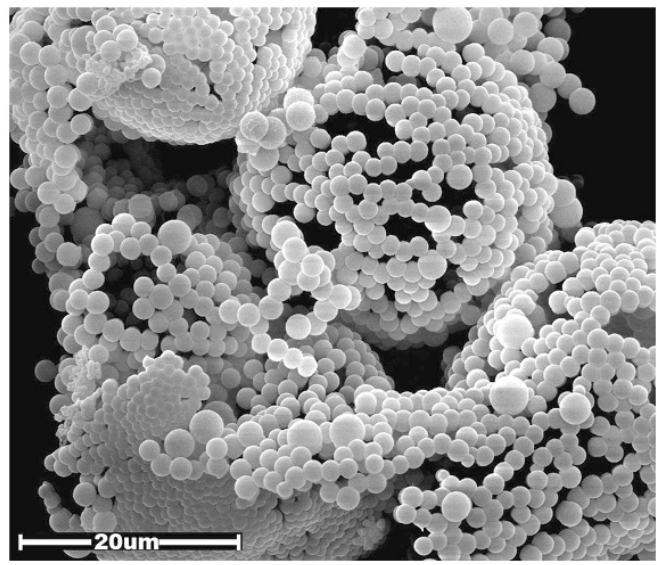

Figure 1 SEM image of Pickering emulsions stabilized by polyazomethine microparticles. 

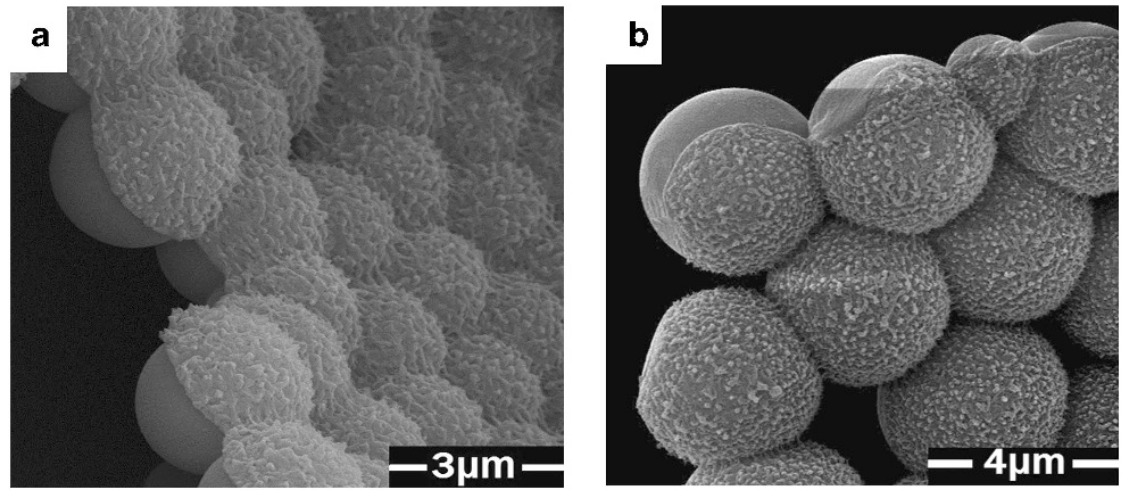

Figure 2 SEM image of polyazomethine anisotropic particles prepared from TPA and TAPP at $120{ }^{\circ} \mathrm{C}$ for $72 \mathrm{~h}$ with concentrations of $2 \%$ (a) and $1 \%$ (b), respectively.
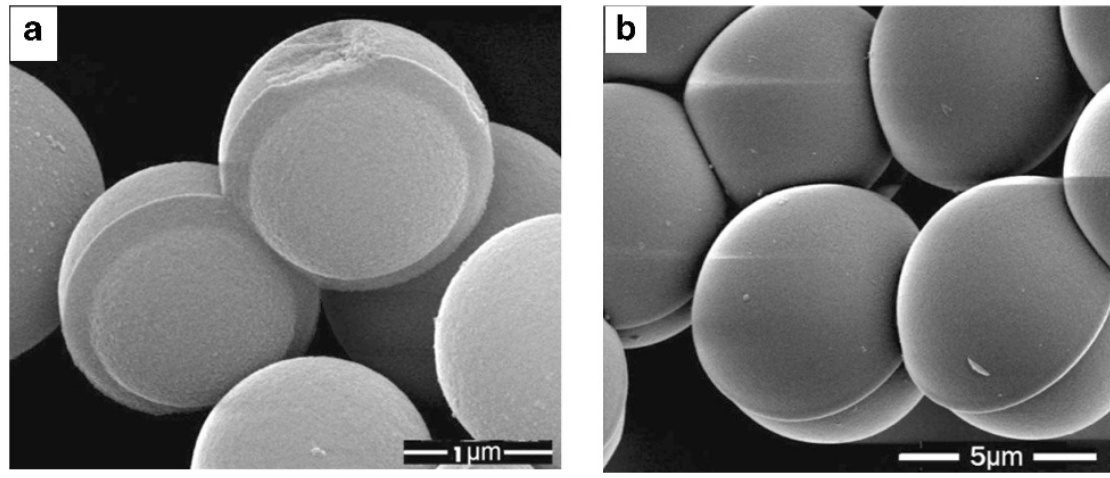

Figure 3 SEM image of polyazomethine anisotropic particles prepared from TPA and tetrakis(4-aminophenyl)porphyrin (a) and TPA and tetrakis (4-aminophenyl)methane (b).
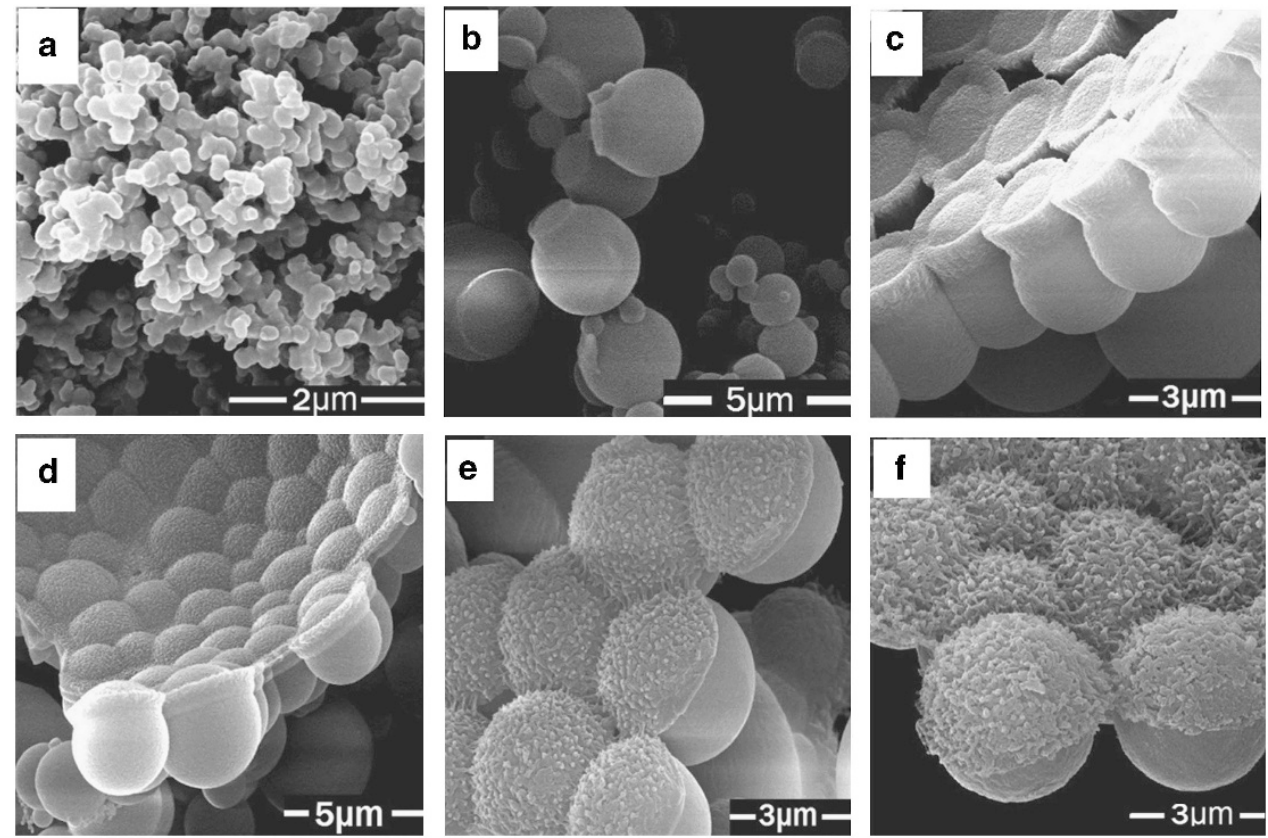

Figure 4 SEM images of polyazomethine anisotropic particles prepared from TPA and TAPP in a solvent mixture of mesitylene/1,4-dioxane/aqueous acetic acid (5/5/1) at different stages: immediately after monomer addition (a), $1.5 \mathrm{~h} \mathrm{(b),} 3 \mathrm{~h}$ (c), $12 \mathrm{~h} \mathrm{(d),} 24 \mathrm{~h} \mathrm{(e)} \mathrm{and} 72 \mathrm{~h}(\mathbf{f})$. 
(Figure $4 \mathrm{~b}$ ). For an additional prolonged solvothermal process, the small remaining amounts of soluble monomers and oligomers precipitated and aggregated on the inner phase of the microsphere due to the reaction-induced self-assembly process, leading to a Christmas ball-like anisotropic structure (Figure 4c). Unexpectedly, this Christmas ball-like anisotropic particle gradually transformed into a semi-hairy anisotropic particle (Figures $4 \mathrm{~d}-\mathrm{f}$ ) with a stable structure that can be maintained throughout the subsequent solvothermal process.

The powder X-ray diffraction patterns revealed that all of the above-mentioned anisotropic particles with different morphologies were amorphous (Supplementary Figure S1), and thus the mechanism behind this continuous morphological transformation is of great interest. Several explanations may help to understand this process. First, because of the limited solubility of aromatic polyazomethines, the initially formed polyazomethine nanoparticles should contain many unreacted amino groups (Supplementary Figure S2), and these amino groups can be protonated by acetic acid so that they can act as polyelectrolyte particle emulsifiers to form a Pickering emulsion. Subsequently, at elevated temperature, the loosely packed nanoparticles will adhere and fuse to form larger, uniform microspheres because of the unique $\mathrm{pH}$-sensitive nature of dynamic imine bonding, which permits the assembly and disassembly of nanostructures. After additional solvothermal treatment, the remaining soluble monomers and oligomers further react and precipitate on the inner phase of the Pickering emulsion template to form a Christmas ball-like anisotropic
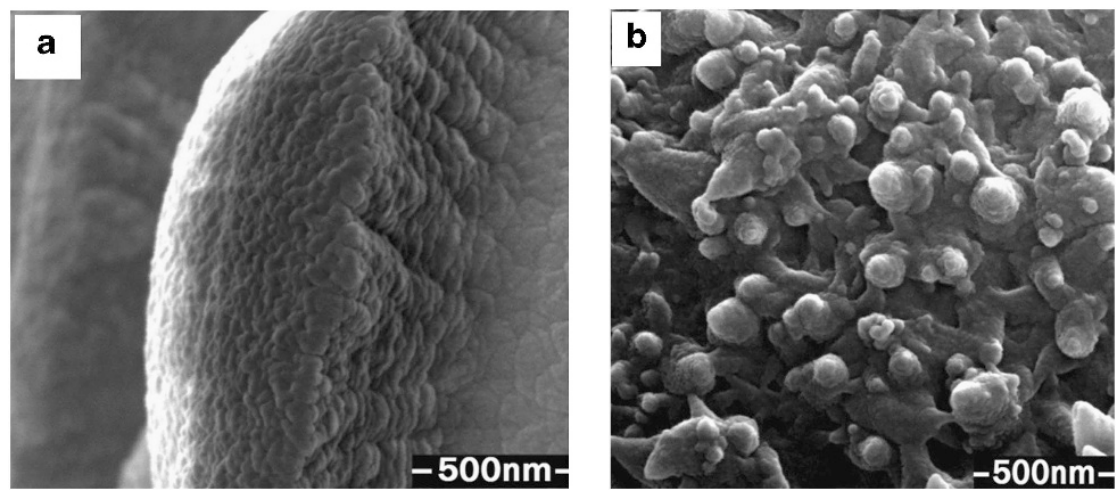

Figure 5 Local SEM images of the Christmas ball-like (a) and semi-hairy (b) polyazomethine anisotropic particles prepared from TPA and TAPP at $120{ }^{\circ} \mathrm{C}$ and $2 \%$ concentration.
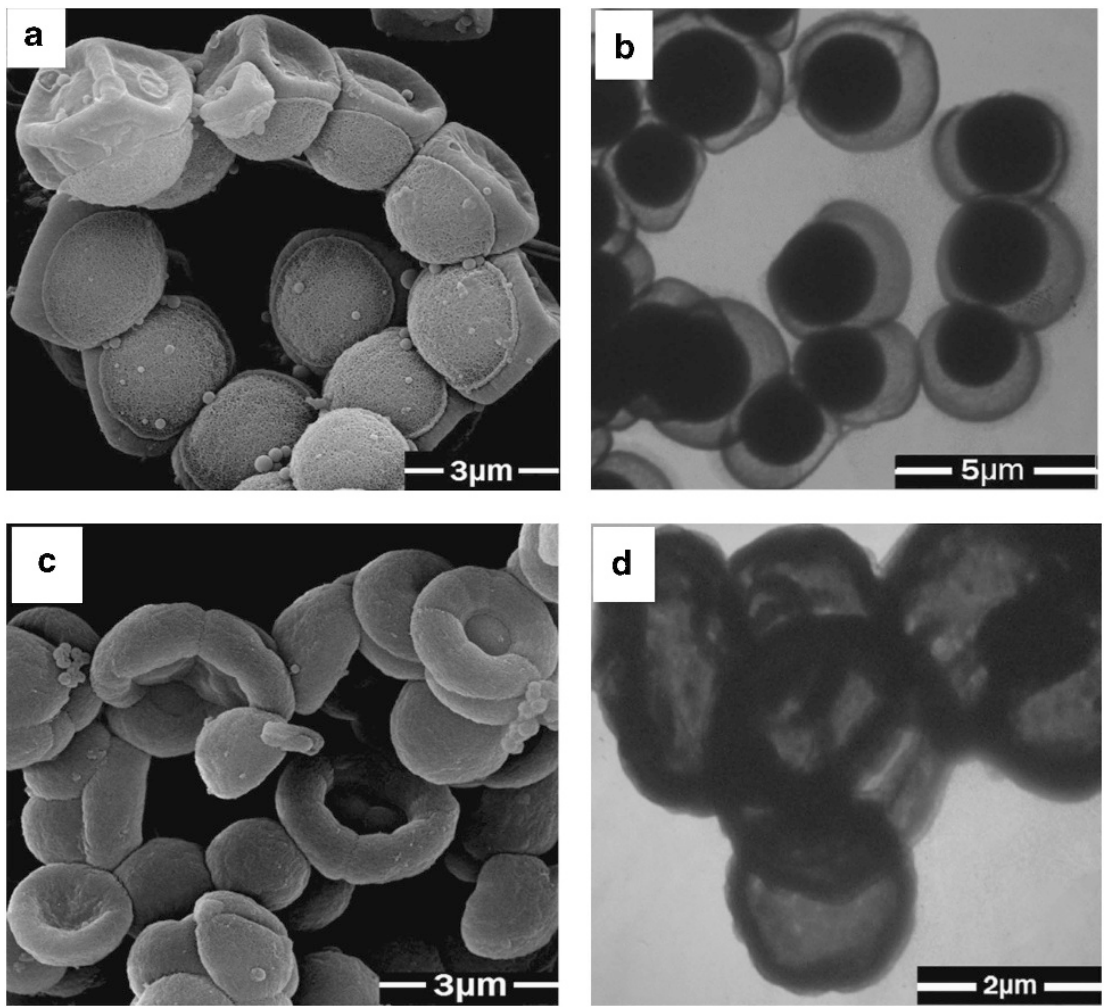

Figure $6 \mathrm{SEM}$ and transmission electron microscopy images of polyazomethine anisotropic particles that were additionally treated in a stronger acid solution (dioxane $10 \mathrm{ml} /$ acetic acid $3 \mathrm{ml}$ ) at $120^{\circ} \mathrm{C}$ for $48 \mathrm{~h}$ (a, b, anisotropic yolk shell) and $96 \mathrm{~h}$ (c, d, anisotropic hollow sphere). 
structure (Figure 4c). The subsequent morphological transformation from Christmas ball-like to semi-hairy anisotropic particles (Figure 4d) is completely unexpected and very difficult to explain. Some recent reports on the selective etching process for the synthesis of inorganic hollow structures may shed some light on this transformation. ${ }^{19-24}$ In particular, Chen and colleagues ${ }^{25}$ demonstrated that the silica nanoparticles formed by a typical Stober method are actually inhomogeneous in nature, and that the inner layer of the shell is chemically less robust than the outer layer and thus can be selectively etched by hot water. A detailed observation of the top of the anisotropic structure reported here reveals that it comprises loosely packed nanoparticles with a relatively smooth and denser shell (Figure 5a); thus, under prolonged treatment, the lessdense nanoparticle aggregates as formed gradually transformed to a sharper and denser nanofiber-like structure (Figure 5b).

If this understanding of the inhomogeneous nature of particles and the corresponding selective etching process accounts for the morphological transformation reported here, then under proper conditions, the polyazomethine anisotropic particle reported here should have a similar hollowing process as that supposed by Chen and colleagues. ${ }^{25}$ To verify this hypothesis, we made a proof-of-principle experiment: the obtained anisotropic particles were additionally treated in a stronger acid solvent to enhance the reversibility of the dynamic imine bonding. After approximately $48 \mathrm{~h}$, SEM revealed that the original solid anisotropic spheres (Supplementary Figure S3) were successfully transformed to yolk-shell structures and, very interestingly, that their anisotropic morphologies were also maintained, which is clearly identified by the SEM images (Figure 6a). The corresponding transmission electron microscopy image showing a solid particle that was encapsulated by a thin shell also clearly confirmed the formation of a complex anisotropic yolk-shell structure (Figure 6b); the chemical structure of polyazomethine yolk shell was also confirmed by Fourier transform infrared spectroscopy (Supplementary Figure S4). Even though there have been several recent reports on the preparation of polymeric yolk-shell microspheres, ${ }^{26,27}$ such a facile template-free approach, according to our knowledge, has not been reported, especially regarding their unique anisotropic morphology. Upon extending the time to $96 \mathrm{~h}$, the yolk-
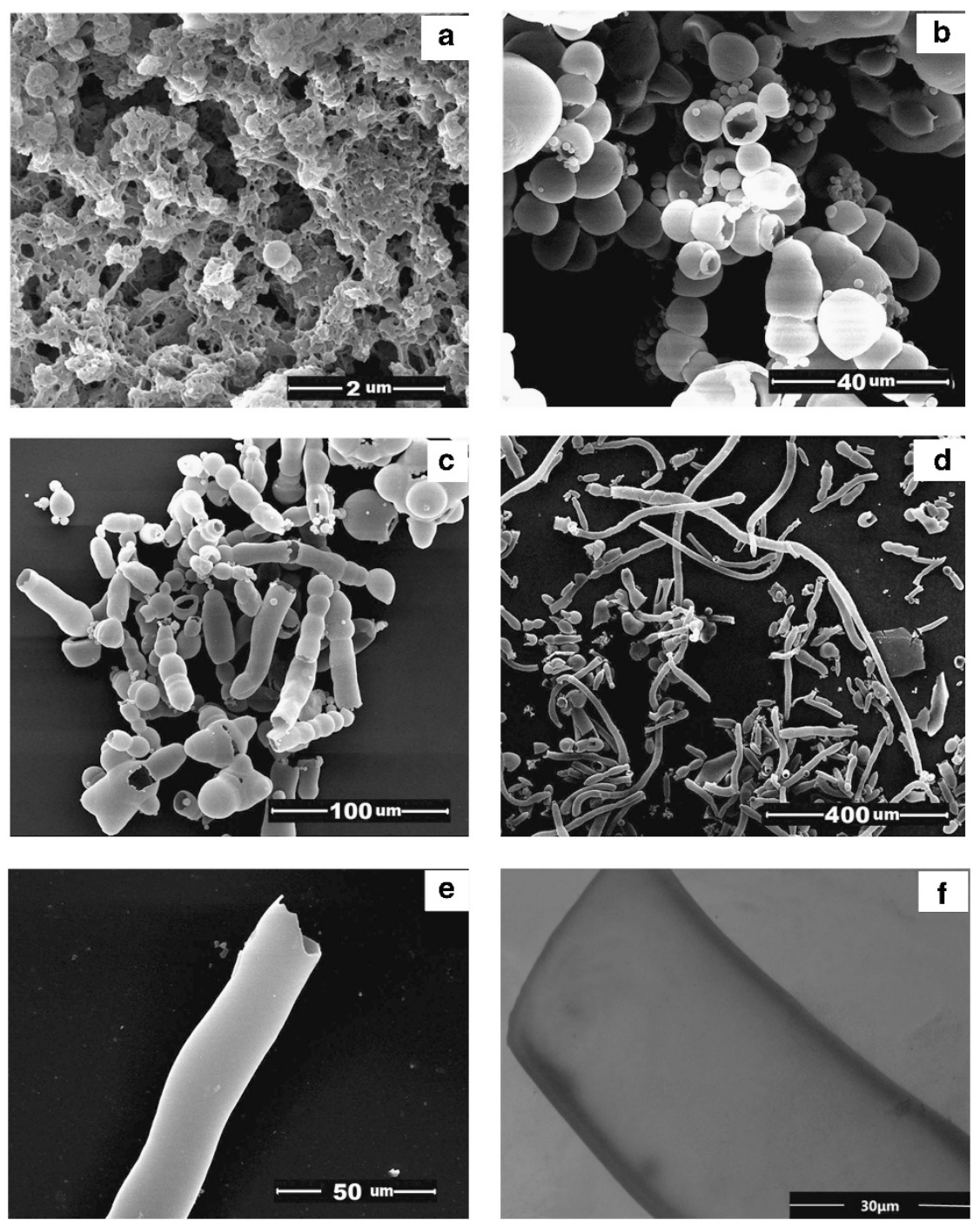

Figure 7 SEM image of polyazomethine hollow spheres and their transformation to microtubes prepared from TPA and TAPP in a solvent mixture of mesitylene/1,4-dioxane/aqueous acetic acid (2.5/7.5/1) at different stages. Morphology transformation of polyazomethine: immediately after monomer addition (a), $1.5 \mathrm{~h} \mathrm{(b),} 7 \mathrm{~h}$ (c), $24 \mathrm{~h}(\mathbf{d})$, enlarged SEM (e) and the transmission electron microscopy image at $24 \mathrm{~h}(\mathbf{f})$. 

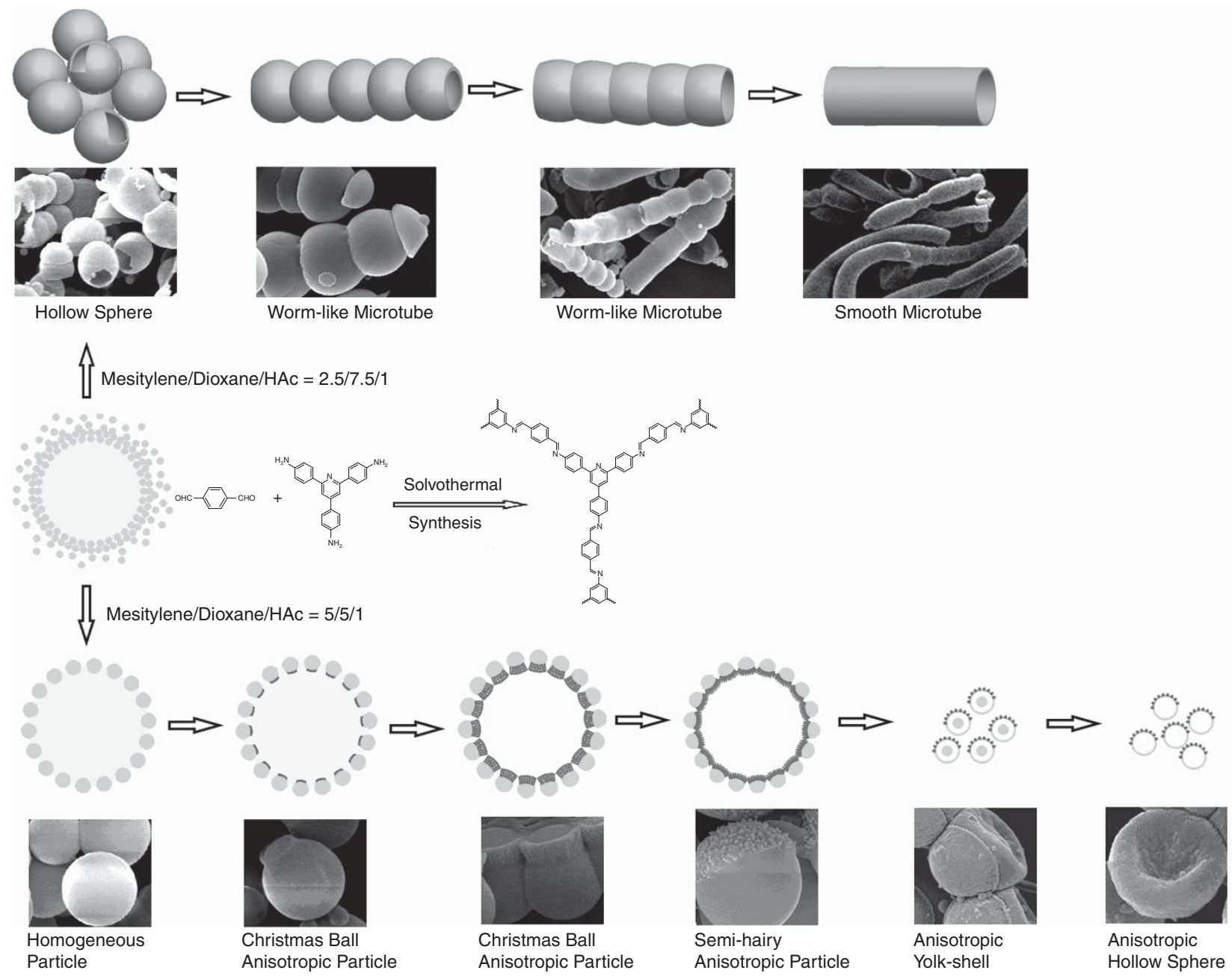

Scheme 1 Schematic presentation of solvothermal synthesis of polyazomethine microspheres by Pickering emulsion templates, and their dynamic imine chemistry-controlled transformation into complex microtubes and anisotropic hollow spheres. A full color version of this figure is available at Polymer Journal online.

shell anisotropic structures that had formed gradually hollowed to a completely anisotropic hollow sphere (Figures $6 \mathrm{c}$ and $\mathrm{d}$ ). This surface to core hollowing process clearly verified that the morphological transformation of anisotropic particles was due to a selective etching process that was enabled by the dynamic nature of imine bonding of polyazomethine and the inhomogeneous nature of the polyazomethine microparticles as formed.

The composition of mesitylene, 1,4-dioxane and aqueous acetic acid is very important for the formation of Pickering emulsions and the corresponding anisotropic structure, so we changed the solution composition to demonstrate the generalizability of this interesting dynamic imine chemistry-enabled morphological transformation. For TPA and TAPP reacted in a mixture solution of mesitylene, 1,4-dioxane and aqueous acetic acid (2.5/7.5/1 vol), polyazomethines also precipitated from the mixture solution, and SEM revealed that the polyazomethines possessed fibrous structures with diameters of approximately tens of nanometers (Figure $7 \mathrm{a}$ ). The polyazomethine fibrous structure as formed can stabilize the immiscible mixtures and form a Pickering emulsion. After solvothermal treatment for $1.5 \mathrm{~h}$, micrometer hollow spheres with smooth surfaces were clearly observed (Figure $7 \mathrm{~b}$ ). Upon extending the solvothermal process to $7 \mathrm{~h}$, the hollow spheres gradually coalesced and transformed to worm- like hollow structures (Figure 7c), and finally to microtubes with smooth surfaces between ten and a hundred micrometers long after $24 \mathrm{~h}$ (Figure $7 \mathrm{~d}$ ). The hollow structures were further confirmed by the transmission electron microscopy image (Figure 7f). This interesting morphological transformation, which has also been seldom reported in polymeric systems, was enabled by the unique $\mathrm{pH}$-sensitive nature of dynamic imine bonding that permitted the assembly and disassembly of polyazomethine nanostructures.

On the basis of the results and analysis above, the solvothermal synthesis of polyazomethine particles and their morphological transformation from irregular nanoparticles to complex anisotropic yolk-shell structures, or the transformation from hollow spheres to worm-like hollow structures and finally to microtubes with smooth surfaces, which were enabled by dynamic imine chemistry, can be depicted as shown in Scheme 1, thus providing a novel template-free approach for the morphology formation and transformation of polymeric materials.

\section{CONCLUSION}

In conclusion, we presented a facile one-pot solvothermal synthesis of anisotropic polyazomethine particles with complex structures using Pickering emulsions formed in situ as templates. A completely 
unexpected continuous morphological transformation from irregular nanoparticles to Christmas ball-like and finally to semi-hairy anisotropic particles was observed when tracing the formation process. The driving mechanism is assumed to be a selective etching process due to the unique $\mathrm{pH}$-sensitive dynamic imine bonding and the inhomogeneous nature of the resulting particles. This argument is supported by the fact that upon additional treatment in a stronger acid to enhance the particles' dynamic nature, the solid anisotropic structures gradually hollowed and transformed to complex anisotropic yolk-shell structures and anisotropic hollow spheres, which have rarely been reported in polymeric systems. The generalizability of this process was further proved by fine tuning the initial solvent composition, through which an interesting morphological transformation from hollow spheres to worm-like tubes and finally to microtubes with smooth surfaces was observed. In the future, though additional verification and development are still necessary, we are convinced that, by judicious selection of the nature of the dynamic covalent bonding, molecular structure of the building blocks and solvothermal conditions, this approach can be a powerful templatefree strategy for the preparation of conjugated polymeric hollow spheres with complex interior structures and functionalities. These studies are currently underway.

\section{ACKNOWLEDGEMENTS}

We are thankful for the financial support provided by the National Natural Science Foundation of China (nos. 20974069 and 21174089), the Ministry of Education (no. JS20091210507067) and Sichuan University (no. 0082204121012)

1 de Gennes, P. G. Soft matter. Rev. Mod. Phys, 64, 645-648 (1992).

2 Nisisako, T., Torii, T., Takahashi, T. \& Takizawa, Y. Synthesis of monodisperse bicolored janus particles with electrical anisotropy using a microfluidic co-flow system. Adv. Mater. 18, 1152-1156 (2006).

3 Du, J. Z. \& O'Reilly, R. K. Anisotropic particles with patchy, multicompartment and Janus architectures: preparation and application. Chem. Soc. Rev. 40, 2402-2416 (2011).

4 Hatton, T. A. \& Lattuada, M. Synthesis, properties and applications of Janus nanoparticles. Nano Today. 6, 286-308 (2011).

5 Walther, A., Hoffmann, M. \& Mueller, A. H. E. Emulsion polymerization using janus particles as stabilizers. Angew. Chem. Int. Ed. 47, 711-714 (2008).

6 Alexeev, A., Uspal, W. E. \& Balazs, A. C. Harnessing Janus nanoparticles to create controllable pores in membranes. ACS Nano 2, 1117-1122 (2008).
7 Liang, F., Liu, J., Zhang, C. L., Qu, X. Z., Li, J. L. \& Yang, Z. Z. Janus hollow spheres by emulsion interfacial self-assembled sol-gel process. Chem. Commun. 47, 1231-1233 (2011).

8 Jiang, S., Chen, Q., Tripathy, M., Luijten, E., Schweizer, K. S. \& Granick, S. Janus particle synthesis and assembly. Adv. Mater. 22, 1060-1071 (2010).

9 Jiang, S., Schultz, M. J., Chen, Q., Mooret, J. S. \& Granick, S. Solvent-free synthesis of Janus colloidal particles. Langmuir 24, 10073-10077 (2008).

10 Hong, L., Jiang, S. \& Granick, S. Simple method to produce Janus colloidal particles in large quantity. Langmuir 22, 9495-9499 (2006).

11 Rowan, S. J., Cantrill, S. J., Cousins, G. R. L., Sanders, J. K. M. M. \& Stoddart, J. F. Dynamic covalent chemistry. Angew. Chem. Int. Ed. 41, 898 (2002).

12 Lehn, J. M. From supramolecular chemistry towards constitutional dynamic chemistry and adaptive chemistry. Chem. Soc. Rev. 36, 151-160 (2007).

13 Maeda, T., Otsuka, H. \& Takahara, A. Dynamic covalent polymers: Reorganizable polymers with dynamic covalent bonds. A. Prog. Polym. Sci. 34, 581-604 (2009).

14 Cote, A. P., Benin, A., Ockwig, N., Matzger, A., O’Keeffe, M. \& Yaghi, O. M. Porous, crystalline, covalent organic frameworks. Science 310, 1166-1170 (2005).

15 El-Kaderi, H. M., Hunt, J. R., Mendoza-Cortes, J. L., Cote, A. P., Taylor, R. E., O'Keeffe, M. \& Yaghi, O. M. Designed synthesis of 3D covalent organic frameworks. Science 316, 268-272 (2007)

16 Uribe-Romo, F. J., Doonan, C. J., Furukawa, H., Oisaki, K. \& Yaghi, O. M. Crystalline covalent organic frameworks with hydrazone linkages. J. Am. Chem. Soc. 133, 11478-11481 (2011).

17 Nishiyabu, R., Teraoka, S., Matsushima, Y. \& Kubo, Y. Fabrication of soft submicrospheres by sequential boronate esterification and their dynamic behavior. ChemPlusChem 77, 201-209 (2012).

18 Yan, Y. Z., Chen, L., Dai, H. J., Chen, Z. H., Li, X. \& Liu, X. K. Morphosynthesis of nanostructured polyazomethines and carbon through constitutional dynamic chemistry controlled reaction induced crystallization process. Polymer (Guildf) 53, 1611-1616 (2012).

19 Zhang, T. R., Ge, J. P., Hu, Y. X., Zhang, Q., Aloni, S. \& Yin, Y. D. Formation of hollow silica colloids through a spontaneous dissolution-regrowth process. Angew. Chem. Int. Ed. 47, 5806-5811 (2008).

20 Zhang, Q., Ge, J. P., Goebl, J., Hu, Y. X., Lu, Z. \& Yin, Y. D. Rattle-type silica colloidal particles prepared by a surface-protected etching process. Nano Res. 2, 583-591 (2009).

21 Zhang, Q., Zhang, T., Ge, J. P. \& Yin, Y. D. Permeable silica shell through surfaceprotected etching. Nano Lett. 8, 2867-2871 (2008).

22 Zhang, Q., Lee, I., Ge, J. P., Zaera, F. \& Yin, Y. Surface-protected etching of mesoporous oxide shells for the stabilization of metal nanocatalysts. Adv. Funct. Mater. 20, 2201-2214 (2010).

23 Park, S. J., Kim, Y. J. \& Park, S. J. Size-dependent shape evolution of silica nanoparticles into hollow structures. Langmuir 24, 12134-12137 (2008).

24 Yu, Q., Wang, P., Hu, S., Hui, J. \& Zhuang, J. Hydrothermal synthesis of hollow silica spheres under acidic conditions. Wang, X. Langmuir 27, 7185-7191 (2011).

25 Wong, Y., Zhu, L., Teo, W., Tan, Y., Yang, Y., Wang, C. \& Chen, H. Y. Revisiting the Stöber method: inhomogeneity in silica shells. J. Am. Chem. Soc. 133, 11422-11425 (2011).

$26 \mathrm{Ji}$, M., Yang, X. \& Wang, J. Synthesis of hollow polymer microspheres with movable polyelectrolyte core and functional groups on the shell-layer. Polymer (Guildf) 50, 5970-5979 (2009).

27 Zhang, M. C., Lan, Y., Wang, D., Yan, R., Wang, S. N., Yang, L. \& Zhang, W. Q. Synthesis of polymeric yolk-shell microspheres by seed emulsion polymerization. Macromolecules 44, 842-847 (2011).

Supplementary Information accompanies the paper on Polymer Journal website (http://www.nature.com/pj) 